\title{
Multipolar Plasmon Resonances in Individual Ag
}

\section{Nanorice}

Hong Wei, $†$ Alejandro Reyes-Coronado, $\neq$ Peter Nordlander, $\mathcal{S}$ Javier Aizpurua ${ }^{\ddagger}$ *,

Hongxing $X u+, \|, *$

Beijing National Laboratory for Condensed Matter Physics and Institute of Physics, Chinese Academy of Sciences, Box 603-146, Beijing 100190, China, Centro Mixto de Física de Materiales CSIC-UPV/EHU and Donostia International Physics Center DIPC, San Sebastián 20018, Spain, Department of Physics and Astronomy, Department of Electrical and Computer Engineering, Laboratory for Nanophotonics, Rice University, Houston, Texas 77005, USA, Division of Solid State Physics/The Nanometer Structure Consortium, Lund University, Box 118, S-22100 Lund, Sweden

*Corresponding authors. E-mail: hxxu@aphy.iphy.ac.cn; aizpurua@ehu.es

† Chinese Academy of Sciences.

‡ CFM CSIC-UPV/EHU and DIPC, San Sebastián.

§Rice University.

" Lund University. 


\begin{abstract}
We study the optical excitation of high-order surface plasmon resonance modes in individual Ag nanorice particles using dark field scattering spectroscopy. We analyse the results by model calculations using the boundary element method. Symmetry breaking caused by oblique illumination makes the even order resonance modes observable in the optical spectrum. All the resonance peaks are found to redshift with increasing length of the particle.
\end{abstract}

Keywords: nanorice, dark-field scattering, multipolar plasmon resonances, oblique excitation

Low dimensional metallic nanostructures have been of considerable interest during the last decade owing to their support of propagating and localized surface plasmon polaritons (SPPs), collective oscillations of electron gas, at the metal-dielectric interfaces. ${ }^{1}$ The localized surface plasmon resonances (LSPRs) in metal nanoparticles (NPs) can result in a drastically enhanced electromagnetic field, which is the physical basis for surface-enhanced Raman scattering (SERS) ${ }^{2-4}$ and fluorescence. ${ }^{5,6}$ The LSPRs of single NPs depend strongly on their size, shape, material, incident excitation and environment, ${ }^{7-9}$ which facilitates their applications in ultrasensitive sensing, ${ }^{10,11}$ surface-enhanced spectroscopy ${ }^{12,13}$ and catalysis. ${ }^{14}$

Among all possible nanoparticle topologies, quasi one-dimensional nanostructures, i.e. nanorods, ${ }^{6,15-20}$ nanorice $^{21}$ and nanowires, ${ }^{22,23}$ have attracted significant interest, due to their highly tunable LSPRs and potential to serve as optical waveguides below the diffraction limit. Previous investigations of these structures have mainly focused on the dipolar resonances in small NPs, while multipolar plasmon resonances have only recently begun to attract attention. Multipolar plasmons in nanorod arrays ${ }^{24}$ and colloidal ensembles ${ }^{25}$ have been investigated by extinction spectroscopy in different sets of nanorods. In those experiments, the separation distance between nanorods was large to avoid interparticle interactions, and different angles of incidence were naturally used on the collections of nanorods, making higher order modes observable, including the usually forbidden even 
modes (forbidden at normal incidence due to symmetry).. More recently, the plasmon modes in individual nanorods and nanowires were studied by photoemission electron microscopy (PEEM) ${ }^{26}$ and aperture-less scanning near field optical microscopy (aSNOM). ${ }^{18,27}$ These investigations have shown the existence of high-order localized modes, which are totally analogous to Fabry-Pérot (FP) modes arising from the reflections between the two ends of the elongated metallic particles.

In this work, we spectroscopically characterize the optical properties of single Ag nanorice particles. Multipolar longitudinal plasmon resonances in individual rice-shaped nanoparticles are detected by dark-field (DF) scattering spectroscopy. With the increase of particle length, the plasmon resonances redshift and higher order resonance peaks appear. The experimental findings are in excellent agreement with theoretical simulations. To the best of our knowledge, it is for the first time that multipolar plasmon resonances are revealed in chemically synthesized single nanoparticles by means of DF scattering spectroscopy.

High throughput synthesis of $\mathrm{Ag}$ nanorice was recently reported, ${ }^{28}$ using poly (vinyl pyrrolidone) (PVP) as surfactant and poly (ethylene glycol) 600 (PEG 600) as reducing agent. The synthesized Ag nanorice particles are centrifuged in ethanol three times to remove the adsorbed chemicals, and finally dispersed in ethanol. Figure 1a shows a typical scanning electron microscopy (SEM) image of the Ag nanorice particle. The average length is about $320 \mathrm{~nm}$ and the width is about $60 \mathrm{~nm}$. The UVVisible-Infrared extinction spectrum of nanorice ensembles in ethanol shown in Figure 1b was measured on a VARIAN Cary5000 spectrometer. A broad absorption peak in near infrared at a wavelength of around $1500 \mathrm{~nm}$ can be easily identified as the longitudinal dipolar resonance. Both non-homogenous broadening from size/shape distribution and radiative decay in each particle contribute to the spectral broadening of the resonance. As will be demonstrated below using simulations, the sharp transverse mode at a wavelength of about $385 \mathrm{~nm}$ is a superposition of several transverse modes and the weak shoulder at a wavelength of about $860 \mathrm{~nm}$ is a mixture of multipolar plasmon resonances of different orders.

The dark-field experiment setup is based on a Leica DM LM microscope and schematically shown in Figure 1c. Non-polarized white light from a halogen lamp was directed from the bottom into a 
transmission type dark-field condenser (Olympus, 1.2-1.4 NA). The light scattered by the sample was collected by a $50 \times$ objective (Leica, 0.75 NA) and directed to a confocal Raman spectroscopy system (Renishaw, inVia) equipped with a 1800-line /mm grating with an air-cooled CCD. Figure 1d shows a typical dark field image for Ag nanorice particles. To study the scattering properties of individual nanorice, a more sparse particle density was used. The nanorice particles in ethanol were dispersed on 3-aminopropyltrimethoxysilane (APTMS) coated ITO glass substrate. ${ }^{29}$ Then, a commercial copper grid (Ted Pella, Inc., Redding, CA) with clear markers is attached on the sample, guaranteeing the direct correlation of dark-field spectra to the SEM images.
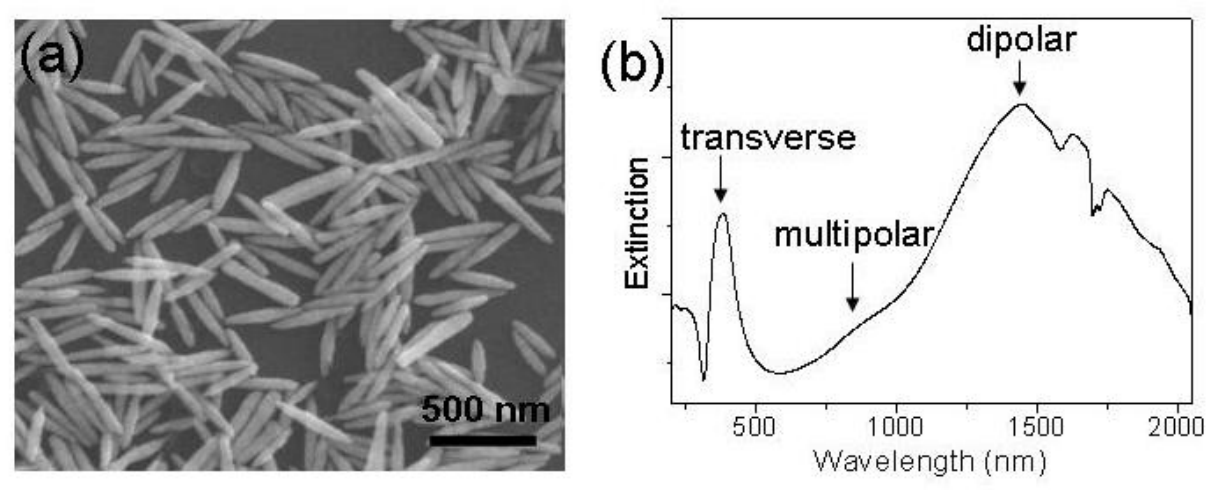

(c)
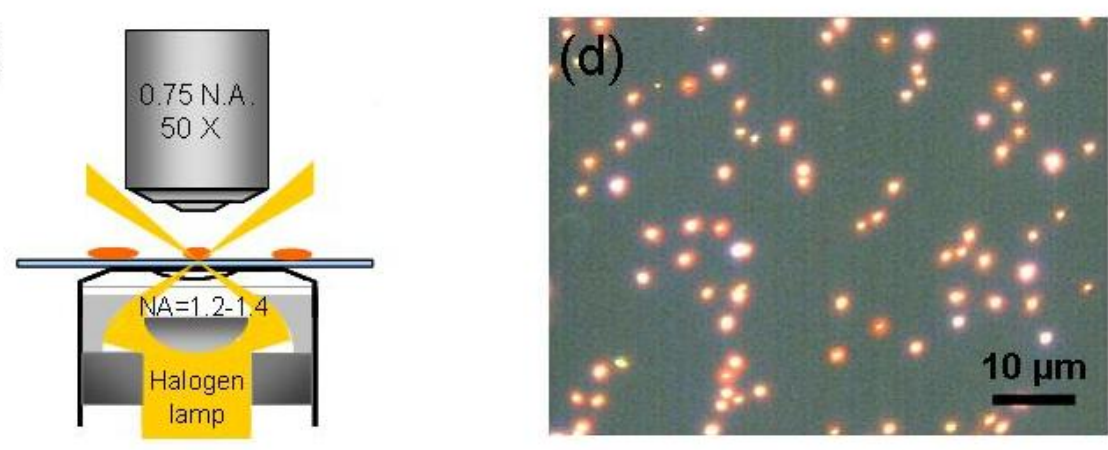

Figure 1. (a) SEM image of Ag nanorice. (b) UV-Visible-infrared spectrum of the nanorice ensemble in ethanol. (c) Schematic drawing of the dark-field setup. (d) Dark field image of Ag nanorice.

To theoretically analyze the experimental results, we perform extensive simulations of the optical extinction cross section of an individual particle with the boundary element method (BEM) ${ }^{30}$ All the 
nanorice were assumed to be perfect spheroids. The propagating direction and polarization of the incident plane wave were both aligned $45^{\circ}$ with respect to the spheroid axis, a configuration that allows the simultaneous excitation of both transverse and longitudinal modes of arbitrary orders. ${ }^{31}$ The spheroid widths were kept constant at $60 \mathrm{~nm}$ in all the calculations. The effect of the substrate was taken into account by assuming an effective index of refraction $n_{\text {eff }}=1.2585$ for the medium surrounding the nanoparticle, obtained by from a simple average of the air and substrate dielectric constants $\varepsilon_{\mathrm{eff}}=\left(\varepsilon_{\mathrm{air}}+\varepsilon_{\mathrm{Glass}}\right) / 2$.

Measured scattering spectra of nanorice of various dimensions and the corresponding SEM images are shown in Figure 2. For small particle sizes, the longitudinal dipolar resonance $(l=1)$ at $\lambda=794 \mathrm{~nm}$ is apparent (see Figure 2a). Similar to single particle studies on small gold nanorods, ${ }^{32,33}$ this dipolar mode exhibit significant radiative broadening. Due to the oblique excitation, symmetry-forbidden modes of even parity become optically active, as labeled $l=2$ and $l=4$ in Figure $2 \mathrm{~b}-\mathrm{d} .^{31}$ With increasing length of the particle, the second order resonance peak grows in amplitude and red-shifts, and the third $(l=3)$ and the fourth $(l=4)$ order longitudinal resonance peaks appear in the spectra (Figure 2c and 2d). In Figure 2e, DF spectra of six nanorice particles of different lengths are shown, in which the redshift of the $l=2$ and $l=3$ resonances can be clearly seen. BEM calculations of the optical extinction in a larger spectral range also show these peak shifts (Supporting Information, Figure S1). 


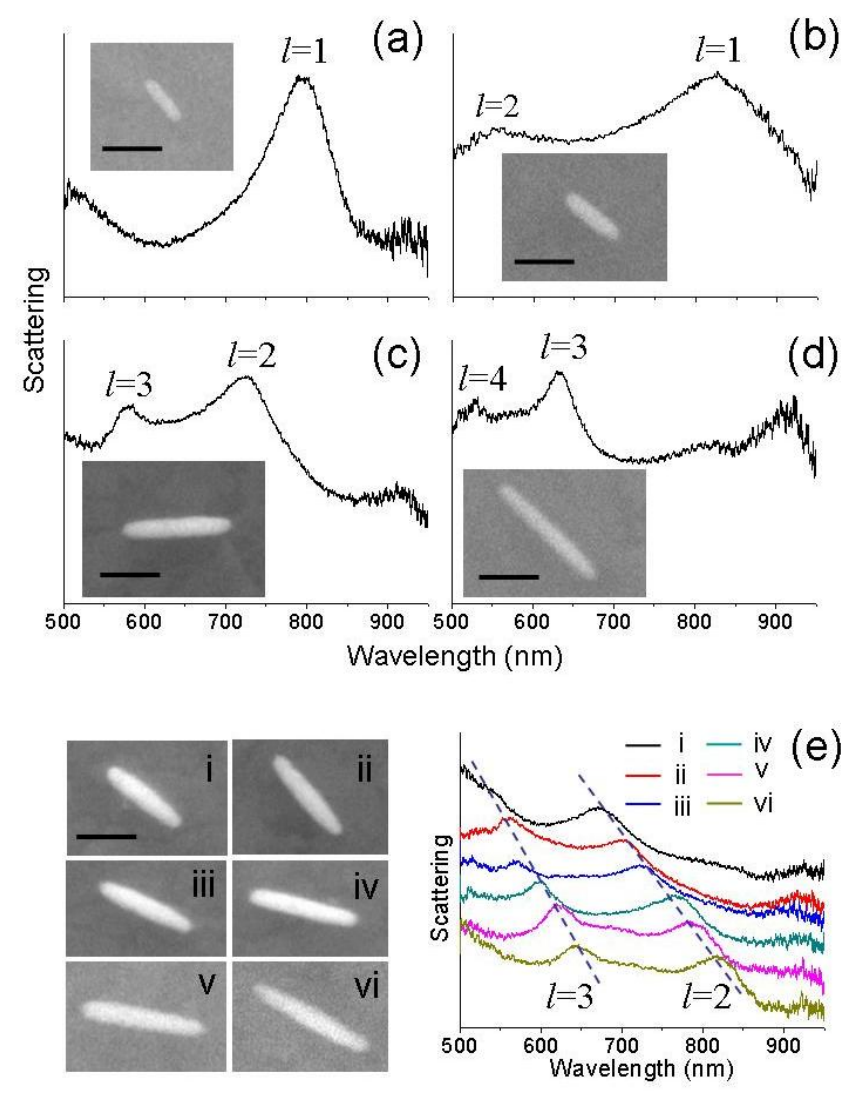

Figure 2. Non-polarized dark field scattering spectra of Ag nanorice of various lengths: (a) $170 \mathrm{~nm}$, (b) $220 \mathrm{~nm}$, (c) $361 \mathrm{~nm}$, (d) $427 \mathrm{~nm}$, (e) $300 \mathrm{~nm}, 328 \mathrm{~nm}, 335 \mathrm{~nm}, 363 \mathrm{~nm}, 417 \mathrm{~nm}, 436 \mathrm{~nm}$ from i to vi, respectively. Longitudinal modes of different orders are well-separated and labeled $l=1-4$ in panel (a) to (d). Corresponding SEM images are shown in the insets. The scale bar is $200 \mathrm{~nm}$. The dash lines in (e) are eye guides.

To make the mode assignments more clear, we plot the length of nanorice versus the experimental peak positions of the optical extinction obtained from DF spectroscopy for hundreds of single nanoparticles, as shown with black points in Figure 3. The experimental dots fall into four distinct bands, divided in the figure by white dashed lines. These bands can be associated to four welldefined orders of plasmon modes, similar to previous studies on rod-shaped particles. ${ }^{24,25}$ A contour plot of the extinction cross-section of $60 \mathrm{~nm}$-wide nanorice of various lengths calculated by BEM is presented as the background of Figure 3. Excellent overall agreement with experimental data for the spectral position of the different orders is obtained. Longitudinal resonant peaks of different orders shift to the red with increasing particle length. The shifting rates decrease with the increase of the order of the resonance. For clarity, the simulation data along the horizontal white dotted line in the 
contour plot is drawn out and plotted as an extinction spectrum corresponding to a nanorice of length equal to $400 \mathrm{~nm}$, which is shown at the bottom of Figure 3.
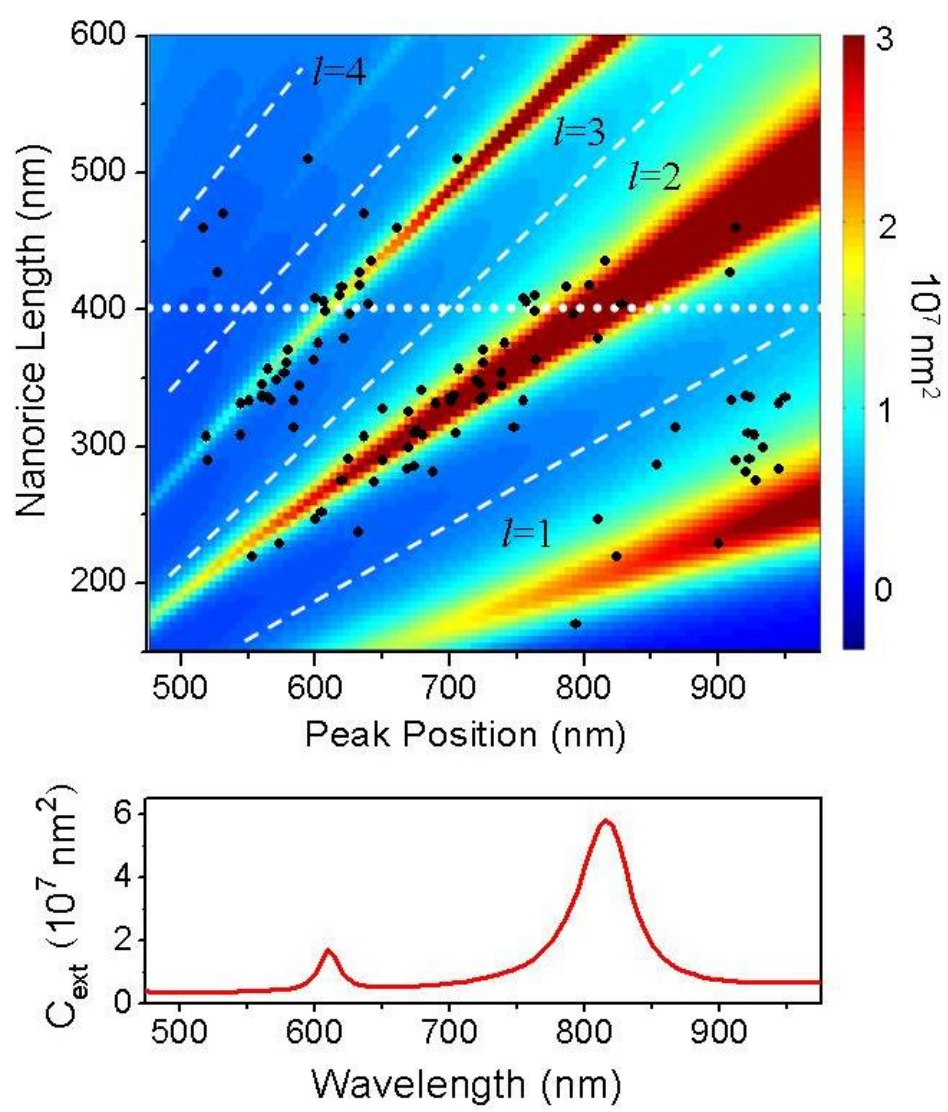

Figure 3. (Top) Dependence of SPR peak positions on nanorice lengths. Black dots: Dark-field scattering peak positions of different resonance orders for nanorice particles of different lengths determined from experiments. Four distinct bands separated by the dashed white lines are apparent, each of which corresponds to a well-defined mode marked from $l=1$ to $l=4$.. Background: Extinction cross-section of individual nanorice (rice width equal to $60 \mathrm{~nm}$ ) calculated from BEM simulations assuming an effective dielectric surrounding, $n_{e f f}=1.2585$. Incident direction and polarization were both aligned $45^{\circ}$ with respect to the symmetry axis of the nanorice. The white dashed lines are plotted for eyeguiding. (Bottom) Extinction coefficient extracted from the white dotted line on the top contour plot for a nanorice with a length of $400 \mathrm{~nm}$. Two modes are clear in the spectrum for this length.

Since the energy of SPRs here lies well below the interband threshold of $\mathrm{Ag}(3.86 \mathrm{eV})$, the damping of multipolar plasmon resonances in Ag nanorice can be attributed primarily to Drude 
damping and radiative damping. As shown in Figure 3, for a nanorice of a given length, lower order modes always present broader peaks and exhibit larger radiative damping. In this case, the nanorice acts as an effective antenna. On the contrary, as opbserved in the contour plot of Fig. 3, higher order modes can serve as high-quality electromagnetic modes thanks to their lower radiative loss. For a given mode order $l$, the intensity of the peak grows with increasing nanorice length, and the radiative loss increases as well. Since the emission power is proportional to the square of the polarization momentum, within the description given by a classical harmonic oscillators, stronger oscillator strength is therefore generated in longer particles. Thus, excitation of higher order modes will lead to higher local field confinement, one primary objective in both solar energy harvesting and enhanced spectroscopy, such as SERS and enhanced fluorescence. ${ }^{34}$

For a better understanding of the multiple SPRs in nanorice, we calculated the electric near-field distributions for a nanorice of $500 \mathrm{~nm}$ length and $60 \mathrm{~nm}$ width. The excitation configuration is shown in Figure 4a, where incident plane wave is at an angle $\theta$ with respect to the main axis of the particle, while linear polarization is kept within the incident plane. The extinction cross-section in Figure $4 \mathrm{~b}$ shows the resonance positions under oblique excitation $\left(\theta=45^{\circ}\right.$ is selected as a representation of the oblique incidence). Five distinct longitudinal resonances at wavelengths $\lambda$ of $1717.5 \mathrm{~nm}, 972.5 \mathrm{~nm}, 715 \mathrm{~nm}, 590 \mathrm{~nm}$ and $519 \mathrm{~nm}$ are obtained in the extinction spectrum, corresponding to the first five multipolar modes. The peaks at $\lambda=362.5 \mathrm{~nm}$ and $\lambda=389 \mathrm{~nm}$ (cyan lines in the left-hand side inset of Figure 4b) are transverse modes, and do not show significant shifts with particle length. It should be noted that under normal incidence $\left(\theta=90^{\circ}\right)$, with polarization aligned along the nanorice axis, only odd modes $(l=1,3,5$, indicated by green lines in Figure $4 \mathrm{~b}$ and the inset) can be excited. Both the longitudinal even modes $(l=2,4$, red lines in Figure $4 \mathrm{~b}$ and the righthand side inset) and transverse modes at $\lambda=362.5 \mathrm{~nm}$ and $\lambda=389 \mathrm{~nm}$ are absent in the spectrum in the case of normal incidence due to symmetry reasons (Supporting Information, Figure S2a). For oblique excitation, the relative intensity of different peaks depends on the angle of incidence (Supporting Information, Figure S2b). In the zoom plot of the transverse modes (left-hand side inset of Fig. 4b), it can be seen that the peak at $\lambda=362.5 \mathrm{~nm}$ is quite broad and looks like a superposition of different 
modes. To get more insights about these transverse modes, we improved the resolution in the simulations and calculated the activation of transverse modes in different excitation configurations (Supporting Information, Figure S3). The results of thesecalculations show that the actual position of the first transverse mode peak is at $\lambda=381.5 \mathrm{~nm}$. The indentification of the nature of the different modes can be easily done by analyzing the near-field distribution around the nanorice. Complementary to the incidence angle shown in Fig. 4b, and since the spectral position of the modes does not change significantly (Supporting Information, Figure S2c), we choose different incident angles to show the near-field distribution of the modes in Fig. 4.c-h. The selected incidence angles (displayed on the top left-hand side of each plot) correspond to cases where the particular mode under study is excited very efficiently, i.e. situations where the strength of a given mode is large. Quasi standing wave patterns are clearly observable in the normalized near field plots of Figure 4c-h. As expected, for normal incidence $\left(\theta=90^{\circ}\right)$ odd-order modes are excited (Fig. 4c,e,g). The o dipolar mode ( $l=1$ in Fig. $4 c)$ presents a node at the center of the particle, while two, three, four and five nodes are present for the second ( $l=2$ in Fig. $4 d)$, third ( $l=3$ in Fig. 4 e), fourth $(l=4$ in Fig. $4 f)$ and fifth $(1=5$ in Fig. $4 \mathrm{~g})$ longitudinal modes respectively. Oblique incidence induced symmetry breaking ensures the excitation of otherwise forbidden modes, (see Figure $4 \mathrm{~d}$ and $4 \mathrm{f}$ for an incidence of $\theta=70^{\circ}$ ). The near-field distribution corresponding to the excitation of a transverse mode is also displayed in Fig. 4h allowing for inmeediate identification of its transverse nature. An important point to consider derived from the near-field distribution calculated around the nanorice, is that electromagnetic energy penetrates drastically into the surrounding medium mainly at the nanoparticle ends enabling possibilities for applications of these particles in sensing. 
(a)

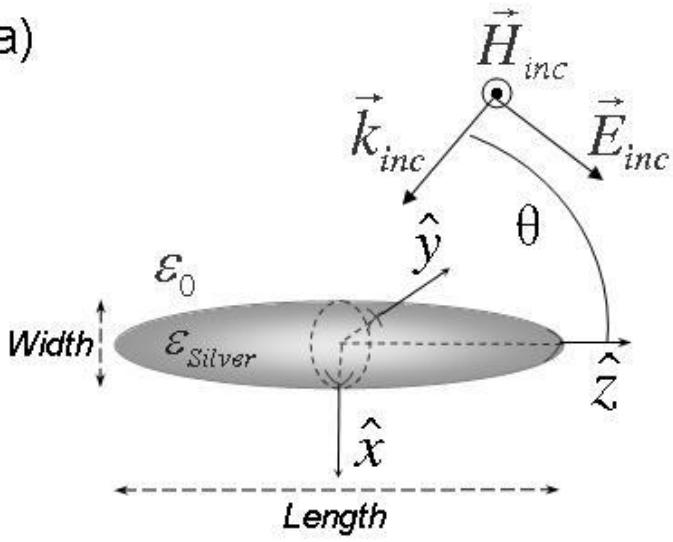

(c)

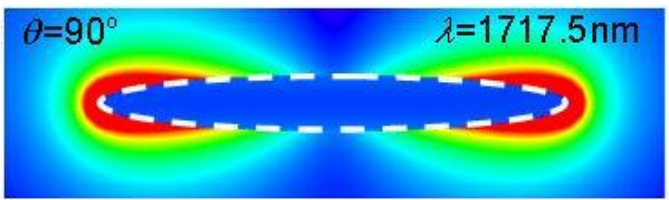

(e)

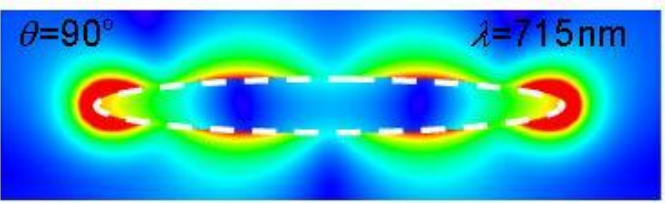

(g)

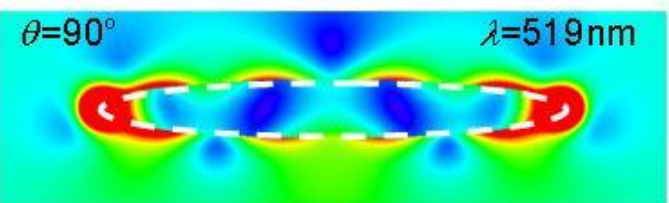

(b)

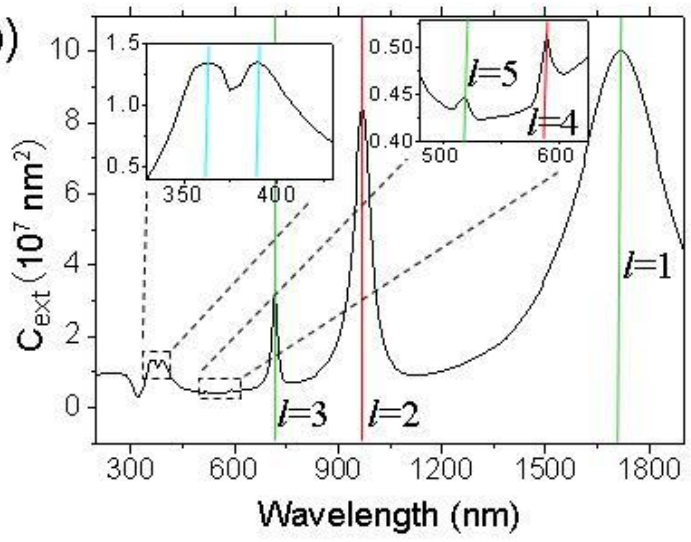

(d)

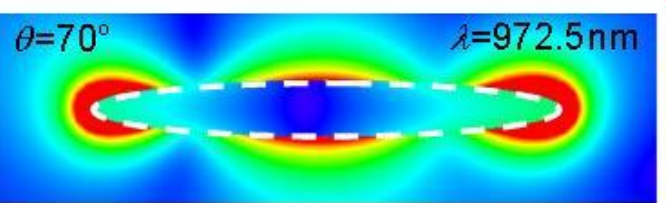

(f)

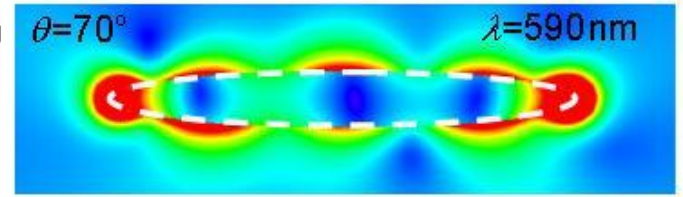

(h)

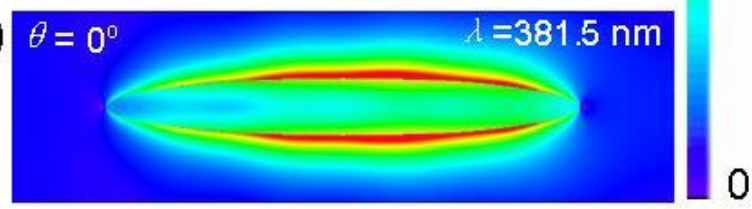

Figure 4. (a) Schematics of the geometry used in the simulations. (b) Simulated extinction cross section for particle of $500 \mathrm{~nm}$ length and $60 \mathrm{~nm}$ width and an incident angle $\theta=45^{\circ}$. The inset to the right-hand side is a zoom of the $4^{\text {th }}$ and $5^{\text {th }}$ longitudinal modes, whereas the inset to the left-hand side is a zoom of the transversal modes. (c-h) Local electric field distribution in the plane $\mathrm{x}-\mathrm{z}$ induced by oblique incidence excitation at different incident angles $\theta$ at the resonant wavelengths $\lambda$ marked in (b). The angles of incidence for the near-field distributions are selected to give emphasis to the particular mode under study.

In summary, we have observed high-order surface plasmon resonance modes in Ag nanorice particles. Recently, some interesting applications based on the elongated taper-ended nanostructures have been theoretically proposed, such as single atom trapping, ${ }^{35}$ metamaterials for optical cloaking $^{36}$ as well as subwavelength color imaging. ${ }^{37} \mathrm{We}$ suggest that Ag nanorice is a highly suitable substrate for LSPR sensing, due to the high sensitivity of the longitudinal resonances on the 
surrounding environments (Supporting Information, Figure S4, S5). For a nanorice of $400 \mathrm{~nm}$ length and $60 \mathrm{~nm}$ width, the peak shifts for the $l=1$ longitudinal mode is $1114 \mathrm{~nm}$ per refractive index unit, which is more sensitive to the environmental refractive index change than that reported in literatures. ${ }^{38-40}$ The multipolar plasmon resonances in the visible to near infrared range also make nanorice particles suitable as substrates in surface-enhanced spectroscopy applications (Supporting Information, Figure S6).

Acknowledgment. We thank Hongyan Liang to supply silver nanorice. We thank Shunping Zhang for helpful discussions and for the help on the manuscript preparation. This work is supported by NSFC Grant No. 10625418, 10874233, MOST Grant No. 2006DFB02020, 2007CB936800, 2009CB930700, "Bairen Project” of CAS. PN acknowledges financial support from The Robert A Welch Foundation, grant C-1222. JA and AR acknowledge financial support from Etortek project inanoGUNE from the Basque Government and project FIS2007-66711-C01-01 from the Spanish Ministry of Science and Innovation.

Supporting Information available: Extinction spectra for different particle lengths, extinction spectra and local electric field distribution of a nanorice excited with different incident angle, transverse resonance modes excited under different incidence configurations, sensitivity of plasmon resonances to the environmental refractive index, and SERS measurements on individual nanorice particles. This material is available free of charge via the Internet at http://pubs.acs.org.

\section{References}

(1) Barnes, W. L.; Dereux, A.; Ebbesen, T. W. Nature 2003, 424, 824-830.

(2) Xu, H. X.; Bjerneld, E. J.; Kall, M.; Borjesson, L. Phys. Rev. Lett. 1999, 83, 4357-4360.

(3) Xu, H. X.; Aizpurua, J.; Kall, M.; Apell, P. Phys. Rev. E 2000, 62, 4318-4324.

(4) Wei, H.; Hao, F.; Huang, Y. Z.; Wang, W. Z.; Nordlander, P.; Xu, H. X. Nano Lett. 2008, 8, 2497-2502. 
(5) Tam, F.; Goodrich, G. P.; Johnson, B. R.; Halas, N. J. Nano Lett. 2007, 7, 496-501.

(6) Aizpurua, J.; Bryant, G. W.; Richter, L. J.; de Abajo, F. J. G.; Kelley, B. K.; Mallouk, T. Phys. Rev. B 2005, 71, 235420.

(7) Kelly, K. L.; Coronado, E.; Zhao, L. L.; Schatz, G. C. J. Phys. Chem. B 2003, 107, 668-677.

(8) Hutter, E.; Fendler, J. H. Adv. Mater. 2004, 16, 1685-1706.

(9) Shuford, K. L.; Lee, J.; Odom, T. W.; Schatz, G. C. J. Phys. Chem. C 2008, 112, 6662-6666.

(10) Willets, K. A.; Van Duyne, R. P. Annu. Rev. Phys. Chem. 2007, 58, 267-297.

(11) Stewart, M. E.; Anderton, C. R.; Thompson, L. B.; Maria, J.; Gray, S. K.; Rogers, J. A.; Nuzzo, R. G. Chem. Rev. 2008, 108, 494-521.

(12) Kneipp, K.; Moskovits, M.; Kneipp, H., Surface-Enhanced Raman Scattering: Physics and Applications. New York, 2006.

(13) Lakowicz, J. R. Anal. Biochem. 2005, 337, 171-194.

(14) Larsson, E. M.; Langhammer, C.; Zoric, I.; Kasemo, B. Science 2009, 326, 1091-1094.

(15) Schider, G.; Krenn, J. R.; Hohenau, A.; Ditlbacher, H.; Leitner, A.; Aussenegg, F. R.; Schaich, W. L.; Puscasu, I.; Monacelli, B.; Boreman, G. Phys. Rev. B 2003, 68, 155427.

(16) Bryant, G. W.; De Abajo, F. J. G.; Aizpurua, J. Nano Lett. 2008, 8, 631-636.

(17) Cubukcu, E.; Capasso, F. Appl. Phys. Lett. 2009, 95, 201101.

(18) Dorfmuller, J.; Vogelgesang, R.; Weitz, R. T.; Rockstuhl, C.; Etrich, C.; Pertsch, T.; Lederer, F.; Kern, K. Nano Lett. 2009, 9, 2372-2377.

(19) Novotny, L. Phys. Rev. Lett. 2007, 98, 266802.

(20) Encina, E. R.; Coronado, E. A. J. Phys. Chem. C 2007, 111, 16796-16801.

(21) Wang, H.; Brandl, D. W.; Le, F.; Nordlander, P.; Halas, N. J. Nano Lett. 2006, 6, 827-832.

(22) Ditlbacher, H.; Hohenau, A.; Wagner, D.; Kreibig, U.; Rogers, M.; Hofer, F.; Aussenegg, F. R.; Krenn, J. R. Phys. Rev. Lett. 2005, 95.

(23) Li, Z. P.; Hao, F.; Huang, Y. Z.; Fang, Y. R.; Nordlander, P.; Xu, H. X. Nano Lett. 2009, 9, 4383-4386.

(24) Krenn, J. R.; Schider, G.; Rechberger, W.; Lamprecht, B.; Leitner, A.; Aussenegg, F. R.; Weeber, J. C. Appl. Phys. Lett. 2000, 77, 3379-3381.

(25) Payne, E. K.; Shuford, K. L.; Park, S.; Schatz, G. C.; Mirkin, C. A. J. Phys. Chem. B 2006, $110,2150-2154$.

(26) Douillard, L.; Charra, F.; Korczak, Z.; Bachelot, R.; Kostcheev, S.; Lerondel, G.; Adam, P. M.; Royer, P. Nano Lett. 2008, 8, 935-940.

Jones, A. C.; Olmon, R. L.; Skrabalak, S. E.; Wiley, B. J.; Xia, Y. N. N.; Raschke, M. B. Nano Lett. 2009, 9, 2553-2558.

(28) Liang, H. Y.; Yang, H. X.; Wang, W. Z.; Li, J. Q.; Xu, H. X. J. Am. Chem. Soc. 2009, 131, 6068-6069.

(29) Xu, H. X.; Kall, M. Chemphyschem 2003, 4, 1001-1005. 
(30) Garcia de Abajo, F. J.; Aizpurua, J. Phys. Rev. B 1997, 56, 15873-15884.

(31) Hao, F.; Larsson, E. M.; Ali, T. A.; Sutherland, D. S.; Nordlander, P. Chem. Phys. Lett. 2008, 458, 262-266.

(32) Sonnichsen, C.; Franzl, T.; Wilk, T.; von Plessen, G.; Feldmann, J.; Wilson, O.; Mulvaney, P. Phys. Rev. Lett. 2002, 88, 077402.

(33) Muskens, O. L.; Bachelier, G.; Del Fatti, N.; Vallee, F.; Brioude, A.; Jiang, X. C.; Pileni, M. P. J. Phys. Chem. C 2008, 112, 8917-8921.

(34) Laurent, G.; Felidj, N.; Aubard, J.; Levi, G.; Krenn, J. R.; Hohenau, A.; Schider, G.; Leitner, A.; Aussenegg, F. R. Phys. Rev. B 2005, 71, 045430.

(35) Chang, D. E.; Thompson, J. D.; Park, H.; Vuletic, V.; Zibrov, A. S.; Zoller, P.; Lukin, M. D. Phys. Rev. Lett. 2009, 103, 123004.

(36) Cai, W. S.; Chettiar, U. K.; Kildishev, A. V.; Shalaev, V. M. Nat. Photonics 2007, 1, 224227.

(37) Kawata, S.; Ono, A.; Verma, P. Nat. Photonics 2008, 2, 438-442.

(38) Lyvers, D. P.; Moon, J. M.; Kildishev, A. V.; Shalaev, V. M.; Wei, A. Acs Nano 2008, 2, 2569-2576.

(39) Lee, K. S.; El-Sayed, M. A. J. Phys. Chem. B 2006, 110, 19220-19225.

(40) Yu, C. X.; Irudayaraj, J. Anal. Chem. 2007, 79, 572-579. 